\title{
Probing the variation of the fine-structure constant using QSO absorption lines
}

\author{
H. Chand ${ }^{1}$, R. Srianand ${ }^{1}$, P. Petitjean ${ }^{2}$ \\ and B. Aracil ${ }^{2} \dagger$ \\ ${ }^{1}$ IUCAA, Post Bag 4, Ganeshkhind, Pune 411 007, India \\ ${ }^{2}$ Institut d'Astrophysique de Paris - CNRS, 98bis Boulevard \\ Arago, F-75014 Paris, France
}

\begin{abstract}
Search for the time variation of the fundamental constants is motivated by various unification theories. Here we present constraints on the variation of the fine-structure constant $\alpha\left(\equiv e^{2} / \hbar c\right)$ obtained using UVES/VLT samples of QSO absorption systems. We find $\langle\Delta \alpha / \alpha\rangle_{w}=(-0.06 \pm 0.06) \times 10^{-5}$ using $23 \mathrm{Mg}$ II systems and the many-multiplet $(\mathrm{MM})$ method. Well selected $15 \mathrm{Si}$ IV systems provide $\langle\Delta \alpha / \alpha\rangle_{w}=(0.15 \pm 0.43) \times 10^{-5}$. Absence of detectable variation in $\alpha$ is also confirmed by our new very high resolution $(R \sim 100,000)$ observation of $z_{\mathrm{abs}}=1.1508$ toward HE $0515-4414$ using HARPS on the ESO 3.6m telescope.
\end{abstract}

\section{Search for $\alpha$ variation using VLT/UVES sample}

Testing the variation of the fundamental constants is important for the understanding of fundamental physics (Uzan et al. 2003). Absorption lines seen in QSO spectra serve as a laboratory to test the variation of $\alpha$ as they allow its measurements at different redshifts (Bahcall et al. 1967). The recently devised many-multiplet method (Dzuba et al. 1999) has been shown to improve the accuracy of $\Delta \alpha / \alpha$ measurements by an order of magnitude (Webb et al. 2001). Based on this method the analysis of a Keck/HIRES sample has resulted in the detection of the variation of $\alpha, \Delta \alpha / \alpha=(-0.54 \pm 0.12) \times 10^{-5}$, over a redshift range of $0.2<z<3.7$ (Murphy et al. 2003). The motivation of our VLT/UVES search is to either confirm or refute the Keck/HIRES result of $\alpha$ variation using high quality data and independent analysis.

Our sample consists of 18 QSO, observed using the UVES on the ESO-VLT Large Programme "QSO absorption lines". The data reduction was performed using the UVES pipeline (Aracil et al. 2004) having typical spectral $\mathrm{S} / \mathrm{N} \sim 60-80, \mathrm{R} \geqslant 44,000$ and wavelength calibration error $\leqslant 3 \mathrm{~m} \AA$ (Chand et al. 2004). Detailed descriptions of our method can be found in Chand et al. (2004). In brief, we used a trial value of $\Delta \alpha / \alpha$ to modify the laboratory wave number $\omega_{0}$ of the multiplets to its rest frame value at $z_{a b s}$, $\omega_{z}$, using $\omega_{z}=\omega_{0}+q\left[(\Delta \alpha / \alpha+1)^{2}-1\right]$, where $q$ is the sensitivity coefficient (Dzuba et al. 1999). Then the absorption systems were modelled for different values of $\Delta \alpha / \alpha$ to get the curve of $\chi_{m i n}^{2}$ as a function of $\Delta \alpha / \alpha$ (Fig. 2 of Chand et al. 2004). From such a curve we derive the best-fit value and error of $\Delta \alpha / \alpha$, using $\chi_{\min }^{2}+1$ statistics.

The simulated data set was used to validate our procedure and devise the proper selection criteria. As a result we avoid (a) blended systems (b) weak $N$ (Fe II) $\leqslant 2 \times 10^{12}$ $\mathrm{cm}^{-2}$ as well as saturated systems. Application of these selection criteria allows us to choose 23 out of a total of $50 \mathrm{Mg}$ II/Fe II systems for the MM method and 15 out of 33 Si IV doublets for the AD method.

$\dagger$ Present address: Department of Astronomy, University of Massachusetts, 710 North Pleasant Street, Amherst, MA 01003-9305, USA. 
Table 1. Summary of recent astrophysical results

\begin{tabular}{|c|c|c|c|}
\hline Species & $z$ & $\Delta \alpha / \alpha / \times 10^{-5}$ & Reference \\
\hline $\begin{array}{c}\text { AD-method } \\
\text { (21 Si IV, HIRES) }\end{array}$ & $2.0-3.0$ & $-0.5 \pm 1.3$ & Murphy et al., 2001, MNRAS, 327, 1237 \\
\hline $\begin{array}{c}\text { MM-method } \\
(128 \text { system, HIRES })\end{array}$ & $0.2-3.7$ & $-0.54 \pm 0.12$ & Murphy et al., 2003, MNRAS, 345, 609 \\
\hline Fe II (1 system, UVES $)$ & 1.1508 & $-0.01 \pm 0.17$ & Quast et al., 2004, A\&A, 415, L7 \\
\hline $\begin{array}{l}\text { MM-method } \\
\text { (23 system, UVES) }\end{array}$ & $0.4-2.3$ & $-0.06 \pm 0.06$ & $\begin{array}{l}\text { Srianand et al., 2004, PRL, } 92121302 \\
\text { Chand et al., 2004, A\&A, 417, } 853\end{array}$ \\
\hline $\begin{array}{c}\text { AD-method } \\
\text { (15 Si IV, UVES) }\end{array}$ & $1.59-2.82$ & $0.15 \pm 0.43$ & Chand et al., 2005, A\&A, 430, 47 \\
\hline Fe II (2 system, UVES $)$ & $1.15,1.84$ & $0.04 \pm 0.15$ & Levshakov et al., 2005, A\&A, in press \\
\hline H I $21-\mathrm{cm}+\mathrm{CO}$ & $0.25,0.69$ & $\leqslant 1.68$ & Carilli et al., 2000, PRL, 85, 5511 \\
\hline H I 21-cm & $\begin{array}{l}0.25 \\
0.69\end{array}$ & $\begin{array}{l}-0.10 \pm 0.22 \\
-0.08 \pm 0.27\end{array}$ & Murphy et al., 2001, MNRAS, 327, 1244 \\
\hline $\mathrm{OH}$ & $\begin{array}{l}0.25 \\
0.25\end{array}$ & $\begin{array}{l}+0.60 \pm 1.00 \\
+0.51 \pm 1.26\end{array}$ & $\begin{array}{l}\text { Kanekar et al., 2004, PRL, 93, } 051302 \\
\text { Darling et al., 2004, ApJ, 612, } 58\end{array}$ \\
\hline O III (73 QSOs) & $0.16-0.80$ & $+7.0 \pm 14.0$ & Bahcall et al., 2004, ApJ, 600, 520 \\
\hline
\end{tabular}

\section{Results and discussion}

Results obtained from the $23 \mathrm{Mg}$ II/Fe II systems using the MM-method and that from the literature are summarised in the upper panel of Fig. 1. The shaded region marks the $3 \sigma$ range around the weighted mean. The simple mean, weighted mean, and standard deviation around the mean obtained for our sample are $(-0.02 \pm 0.10) \times 10^{-5}$, $(-0.06 \pm 0.06) \times 10^{-5}$, and $0.41 \times 10^{-5}$ respectively (Chand et al. 2004; Srianand et al. 2004). The $3 \sigma$ constraint corresponds to $-0.24 \leqslant \Delta \alpha / \alpha$ (in $\left.10^{-5}\right) \leqslant+0.12$ over the redshift range of $0.4 \leqslant z \leqslant 2.3$. The distribution of our 15 measurements using $\mathrm{Si}$ IV is shown in the lower panel of Fig. 1. For comparison on the same scale, the measurements using the MM method are also shown. The weighted mean value obtained from the ADmethod over the redshift range $1.59 \leqslant z \leqslant 2.82$ is $\Delta \alpha / \alpha=(0.15 \pm 0.43) \times 10^{-5}$. The $3 \sigma$ range $\left(-1.14 \times 10^{-5} \leqslant \Delta \alpha / \alpha \leqslant 1.44 \times 10^{-5}\right)$ is shown by the wider shaded region.

In addition we have also carried out our study by using very high resolution spectrum ( $R \sim 100,000)$ of HE 0515-1444 observed using HARPS, by analysing its absorption system at $z_{\mathrm{abs}}=1.1508$. The preliminary result, $\Delta \alpha / \alpha=(-0.10 \pm 0.25) \times 10^{-5}$ was obtained using only Fe II multiplets as is shown by an asterisk $\left(^{*}\right)$ in the upper panel of Fig. 1, is also found to be consistent with our UVES samples results of no detectable variation in $\alpha$ (Chand et al. 2005, in preparation).

From Table 1, that summarises all the recent measurements by different methods and/or by independent analysis, it is evident that, except for the MM-method result of HIRES sample, there is no evidence for time variation of $\alpha$. The increased accuracy in our result from the UVES sample is mainly due to the better quality of the data and using the proper sample selection criteria.

\section{Acknowledgements}

Based on observations collected at the European Southern Observatory (ESO), under the Large Programme ID No. 166.A-0106 with UVES on the 8.2m Kuyen telescope operated at the Paranal Observatory, Chile. HC thanks CSIR, INDIA for the grant award No. 9/545(18)/2KI/EMR-I. We gratefully acknowledge support from the Indo-French 

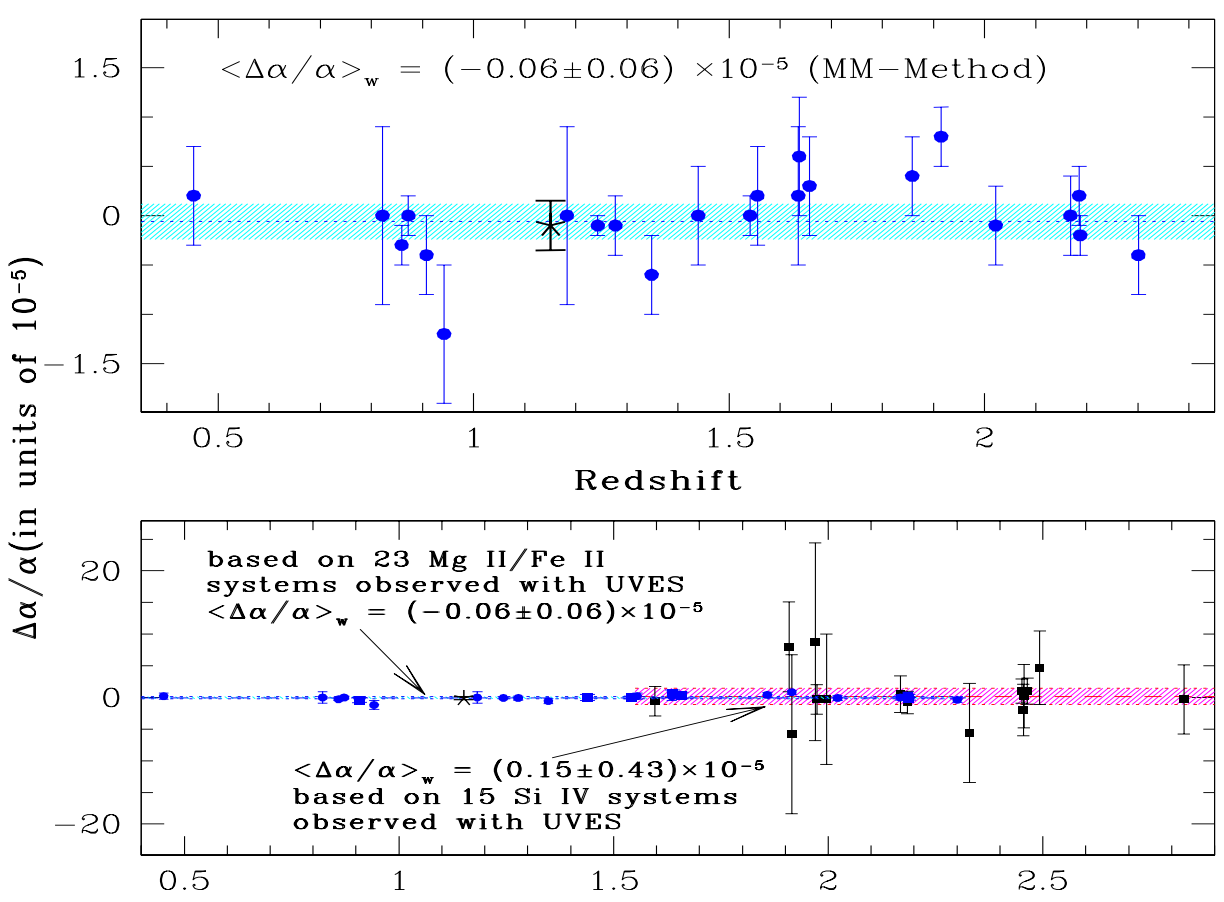

Redshift

Figure 1. In the upper panel the results from the MM-method (using $23 \mathrm{Mg}$ II/Fe II systems) are shown by filled circle. Shadow regions mark the $3 \sigma$ range around the weighted mean. An asterisk $\left({ }^{*}\right)$ shows our preliminary result based on the analysis of Fe II multiplets in $R \sim 100,000$ spectrum of HE 0515-1444, obtained using HARPS. In the lower panel solid squares show the measurements using Si IV doublets (AD method). The measurements using the MM-method are also shown in this panel for comparison, by filled circles and the narrow shaded region.

Centre for the Promotion of Advanced Research (Centre Franco-Indien pour la Promotion de la Recherche Avancée) under contract No. 3004-3.

\section{References}

Aracil, B., Petitjean, P., Pichon, C., Bergeron, J., 2004, A\&A, 419, 811

Bahcall, J. N., Sargent, W. L. W., Schmidt, M., 1967, ApJ, 149, L11

Chand, H., Petitjean, P., Srianand, R., Aracil, B., 2005, A\&A, 430, 47

Chand, H., Srianand, R., Petitjean, P., Aracil, B., 2004, A\&A, 417, 853

Dzuba, V. A., Flambaum, V. V., Webb, J. K., 1999, Phys. Rev. Lett., 82, 888

Murphy, M. T., Webb, J. K., Flambaum, V. V., 2003, MNRAS, 345, 609

Murphy, M. T., Webb, J., Flambaum, V., et al., 2001, MNRAS, 327, 1237

Quast, R., Reimers, D., Levshakov, S. A., 2004, A\&A, 415, L7

Srianand, R., et al., 2004, Phys. Rev. Lett., 2004, 92121302

Uzan, J., 2003, Rev. Mod. Phys., 75, 403

Webb, J. K., Murphy, M. T., Flambaum, V. V., et al., 2001, Phys. Rev. Lett., 87, 091301 